\title{
Editorial
}

\section{Corona-Krise als Herausforderung für das Bildungswesen. Perspektiven aus Sicht der Bildungswissenschaften}

\author{
Stephanie Moser ${ }^{1}$, Doris Lewalter ${ }^{1}$, Henrik Saalbach ${ }^{2,3}$, \\ Olaf Köller 4 \& Sabine Walper ${ }^{5}$ \\ Technische Universität München \\ Leipziger Forschungszentrum für frühkindliche Entwicklung, LFE \\ Universität Leipzig \\ 4 Leibniz-Institut für die Pädagogik der Naturwissenschaften und Mathematik, Kiel \\ Deutsches Jugendinstitut, München
}

Die weitreichenden Kontaktbeschränkungen in den pandemiebedingten Lockdowns 2020 und 2021 führten zu einer immensen Veränderung unseres gewohnten Alltags. Besonders massiv wirkten sich die Einschränkungen auf den Betreuungs- und Bildungssektor aus: Kindertagesstätten, Schulen und Hochschulen waren innerhalb kürzester Zeit von umfassenden Schließungen betroffen. Dies führte dazu, dass sich Familien, Lernende, Betreuungs- und Lehrpersonal in rasanter Geschwindigkeit mit den in ihrer Region geltenden Richtlinien arrangieren und sich den jeweiligen Umständen anpassen mussten. Die Ausgestaltung der institutionellen Betreuungsund Bildungsangebote fiel sehr unterschiedlich aus, von reiner Notbetreuung in Kitas und Schulen über Wechselunterricht bis hin zu Distanzunterricht, welcher in unterschiedlichster Form synchron und asynchron dargeboten wurde. Die direkten Auswirkungen waren für viele belastend: jüngere Kinder mussten von den oftmals gleichzeitig erwerbstätigen Eltern nun rund um die Uhr zu Hause betreut werden, Homeschooling musste ermöglicht und begleitet werden, die Schüler*innen und Studierenden mussten beim Lernen selbstgesteuert auf digitale Lösungen zurückgreifen - und alle Beteiligten mussten innerhalb ihrer Möglichkeiten versuchen, die stark eingeschränkten sozialen Kontakte zu kompensieren. Alles zusammen führte zu einer außergewöhnlichen Belastungs- und Ausnahmesituation, die auch in der Bildungslandschaft bislang so noch nicht erlebt wurde.

Psychologie in Erziehung und Unterricht, 2021, 68, 267-270

DOI 10.2378/peu2021.art21d

(c) Ernst Reinhardt Verlag München Basel
Eine Aufarbeitung dieser Ereignisse und der langfristigen Folgen der Pandemie ist aus der Sicht der Bildungsforschung unumgänglich. Der vorliegende Corona-Sonderteil der PEU sammelt daher verschiedene Beiträge im Forumsformat, welche die Situation der Bildungslandschaft während der Corona-Pandemie und deren Auswirkungen aus Sicht der Bildungswissenschaften thematisieren: die beteiligten Wissenschaftler*innen berichten über deutschlandweite Befragungen sowie über spezifischere Erhebungen in einzelnen Bundesländern und über die Situation in Österreich. Für ein umfassendes Bild wurden dabei die Sichtweisen sowohl von Schüler*innen und Studierenden als auch von Eltern, frühpädagogischen Fachkräften und Lehrenden einbezogen.

In den Beiträgen wurde grundlegend den folgenden Fragen nachgegangen: Unter welchen Bedingungen fand Betreuung und Lernen während der Corona-Krise statt? Welche Konsequenzen hatten die massiven Einschränkungen zur Bekämpfung der Pandemie? Wie lassen sich negative Folgen kompensieren und wie kann man institutionelle Betreuungs- und Bildungseinrichtungen in Zukunft besser aufstellen und vorbereiten?

Im ersten Beitrag beleuchtet Yvonne Anders die Situation jüngerer Kinder in deutschen Kindertagesstätten. Die Autorin zeigt für Kinder im Krippen- und Kindergartenalter auf, dass neben der Problematik der fehlenden Betreuungsmöglichkeiten während der Pandemie auch der Ent- 
zug der frühpädagogischen Bildungsangebote beachtet werden muss. Die ersatzweise durchgeführten digitalen Bildungs- und Kontaktangebote greifen für die Kleinsten oftmals nicht in dem Maße wie für ältere Kinder. Gleichzeitig geht mit elterlicher Erwerbstätigkeit und zeitgleicher Betreuung von Kleinkindern ein erhöhtes Belastungserleben für Familien einher. Die Autorin mahnt an, dass die negativen Folgen der Pandemie (etwaige Entwicklungsrückstände) durch entsprechende Maßnahmen abzufedern, etwaige positive Veränderungen (z. B. innovative digitale Konzepte in der Zusammenarbeit mit Eltern) aber beizubehalten sind.

Thorsten Naab und Alexandra N. Langmeyer nehmen die Kindergartenkinder in Deutschland in den Blick. Sie berichten über Forschungsergebnisse, die sich mit der Bedeutung von institutionellen Bildungseinrichtungen für Freundschaftskontakte und dem daraus resultierenden Wohlbefinden der Kinder beschäftigen. Die Autor*innen legen u. a. dar, dass die reduzierten Kontakte zu Gleichaltrigen während des ersten Lockdowns gerade bei den jüngeren Kindern nicht durch Notbetreuung oder medial gepflegte Kontakte ersetzt werden konnten. Weiterhin kommen sie zu dem Schluss, dass Bildungs- oder finanzielle Benachteiligung mit einer im Vergleich eingeschränkteren Kontaktpflege einhergeht. In der Konsequenz betonen die Autor*innen die zentrale Rolle von institutionellen Bildungseinrichtungen für die soziale Entwicklung und das kindliche Wohlbefinden.

Zwei Beiträge widmen sich spezifisch der Lage jüngerer Schüler*innen, indem Grundschüler*innen in Deutschland (Gunzenhauser und Saalbach) sowie in Österreich (Weber, Helm \& Kemethofer) in den Blick genommen werden.

Catherine Gunzenhauser und Henrik Saalbach analysieren auf der Basis von Elternbefragungen die Lernbedingungen während der Pandemie an deutschen Grundschulen. Rückgreifend auf das Angebot-Nutzungsmodell (Helmke, 2015) werden daraus Konsequenzen für die Kompetenzentwicklung der Schüler*innen ge- zogen. Die Autor*innen argumentieren, dass auf Angebotsseite weniger kognitive Aktivierung und weniger Feedback während der Pandemie negative Auswirkungen auf die Leistung der Schüler*innen haben könnte, was sich insbesondere auch für die Bewertung von Übertrittentscheidungen als problematisch erweisen könnte. Hinsichtlich der Wirkungsseite fordern sie weitere Lernstanderhebungen zur objektiven Bewertung der Lernleistung während der Corona-Krise.

Christoph Weber, Christoph Helm und David Kemethofer beschreiben anhand von Lehrkräfte- und Elternbefragungen die Ausprägungen des Distanzlernens der österreichischen Grundschüler*innen. Dabei wird deutlich, dass der gerade für die jüngeren Schüler*innen wichtige synchrone Online-Unterricht nur in unzureichendem Ausmaß stattfand. Sie erläutern weiterhin, dass vor allem die jüngeren Schüler*innen auf die elterliche Unterstützung im Homeschooling angewiesen waren, insbesondere bei selbstgesteuerten asynchronen Lernphasen. Die Autoren schlussfolgern, dass die Ausgestaltung von Distanzunterricht in der Grundschule genauer in den Blick genommen werden sollte sowie dass die Kommunikation zwischen Eltern und Lehrkräften gerade für Grundschulkinder zentral für erfolgreiches Lernen im Distanzunterricht angesehen werden kann.

Der Beitrag von Birgit Lütje-Klose, Sabine Geist und Janka Goldan thematisiert Schüler*innen mit sonderpädagogischem Förderbedarf und analysiert die spezifischen Herausforderungen, denen diese Schüler*innen während der Corona-Krise in Deutschland ausgesetzt waren. Die Autorinnen berichten, dass sich insbesondere für Schüler*innen mit sonderpädagogischem Förderbedarf aus der Pandemiesituation gravierende Nachteile ergaben. Sie beschreiben darüber hinaus Möglichkeiten zur Förderung dieser Schüler*innen während des Distanzunterrichts. Schließlich zeigen sie, dass gerade die persönliche Beziehung zur Lehrkraft und die soziale Schulgesellschaft für diese Schüler*innen von großer Bedeutung für ihr Lernen sind. 
Ludger Wößmann und Larissa Zierow berichten über die Ergebnisse zweier Elternbefragungen zur schulartübergreifenden Situation von Schulkindern in Deutschland während der Coronapandemie. Die Autor*innen berichten auf der Basis verschiedener Zeitnutzungsstudien, dass die Lernzeiten während der Pandemie im Vergleich zu vorher deutlich gesunken sind. Sie erläutern darüber hinaus die Aktivitäten der Schulen und die ergriffenen zusätzlichen Fördermaßnahmen. Die Autor*innen beschreiben weiterhin die Erfahrungen der Eltern im Homeschooling und berichten über deren Einschätzung von negativen aber auch positiven Auswirkungen des Distanzunterrichts auf das Lernen und Leben ihrer Kinder.

Christoph Helm und Alexandra Postlbauer berichten ebenfalls mittels Elternbefragungen über die schulartübergreifende Lage der Schüler*innen in Österreich. Darüber hinaus vergleichen sie relevante Bildungsstudien zum Lockdown für Österreich, Schweiz und Deutschland. Sie zeigen beispielsweise, dass die Belastung der Schüler*innen und ihrer Eltern während des letzten Jahres deutlich gestiegen ist. Die Autor*innen fordern ebenfalls bezüglich der Einschätzung des Kompetenzerwerbs objektive Lernstanderhebungen und Leistungsdaten sowie Fördermaßnahmen insbesondere für benachteilige Schüler*innen.

Die Auswirkungen der Corona-Pandemie auf die Situation von Studierenden in Deutschland werden von Markus Lörz, Lena Maria Zimmer und Jonas Koopmann in den Blick genommen. Während die Umstellung auf den komplett digitalen Betrieb an den Hochschulen zwar schnell gelungen ist, berichten die Autor*innen mittels Studierendenbefragungen von den Herausforderungen, die damit einhergehen. Der fehlende Kontakt zu den Lehrenden und Kommiliton*innen und die oftmals verschlechterte finanzielle Lage werden von den Studierenden als zentrale Belastung empfunden. Das Studienabbruchrisiko ist dabei im Vergleich zu Zeitpunkten vor der Pandemie gestiegen und für bestimmte vulnerable Studierendengruppen besonders hoch. Die Autor*innen regen etwa finanzielle Unterstützungshilfen für Studierende und Lernunterstützung im Falle weiterer digitaler Semester an.

Als Fazit aller Beiträge zeigt sich vor allem, dass die bereits seit Langem bestehende Chancenungleichheit im institutionellen Bildungssysstem durch die Maßnahmen zur Pandemiebekämpfung nochmals verschärft wurde. Insbesondere schon vorher benachteiligte Kinder, Schüler*innen, Studierende und ihre Familien sind auch jetzt immens von den Betreuungs- und Unterrichtsausfällen sowie den Kontaktbeschränkungen negativ betroffen.

Weiterhin verdeutlichen die Berichte der Wissenschaftler*innen: insbesondere die jüngeren Kinder und Schüler*innen sind erkennbar stärker von den Maßnahmen beeinträchtigt und auch stärker abhängig von der Betreuung und Hilfe im Elternhaus (gerade bei medial gepflegten Kontakten oder in asynchronen Lernsituationen!). Die Konsequenz, die sich hier ziehen lässt, ist u. a., dass die Eltern deutlich stärker unterstützt werden müssen, sowohl bei der Vereinbarung von Erwerbstätigkeit und Betreuung als auch darin, wie sie wiederum das Lernen ihrer Kinder begleiten und fördern können.

Aber es zeigt sich auch, dass es in den Erfahrungen, die während der Pandemie gemacht wurden, eine große Varianz gibt: die Wissenschaftler*innen berichten auch Befunde zu positiven Erfahrungen im Lockdown, etwa davon, dass die Lernenden die erhöhte Selbststeuerung des digitalen Lernens als Erleichterung empfinden. Dies ist ein klarer Hinweis, auf mehr Autonomie und Individualisierung auch im Präsenzunterricht zu achten. Die abrupte Umstellung auf online-Formate während der Corona-Krise kann weiterhin als dringend notwendiger Digitalisierungsschub gesehen werden, wobei innovative und erfolgreiche Konzepte beibehalten werden sollten.

Die mittel- bis langfristigen Forderungen der Autor*innen in diesem Corona-Sonderteil gehen vorwiegend in zwei Richtungen: zum einen wird die Forderung nach umfassenden Leistungserhebungen laut, um sich mittels objektiver Leistungsdaten einen Überblick über die tatsächlichen Lernlücken und daraus zu 
ziehenden Konsequenzen zu verschaffen. Bisher ist die Datenlage hierzu noch unzureichend. Zum anderen werden Forderungen nach gezielten Fördermaßnahmen gestellt, um die Schulschließungen zu kompensieren und eventuell vorhandene Lernlücken aufzuholen. Schließlich müssen im Rahmen der Digitalisierung umfassende Konzepte entwickelt werden, die nicht nur für eine ausreichende technische Ausstattung aller Lernenden und Lehrenden sorgen, sondern auch didaktisch sinnvolle Ansätze digitaler Kommunikation und Lehre den Erziehenden und Lehrenden (etwa über Fortbildungsmaßnahmen) vermitteln.

Unser Dank gilt allen Autor*innen, die mit ihren Einschätzungen zu einem umfassenden Überblick über die Corona-bedingten Herausforderungen für das Bildungswesen beigetragen haben.

\section{Literatur}

Helmke, A. (2015). Unterrichtsqualität und Lehrerprofessionalität. Diagnose, Evaluation und Verbesserung des Unterrichts. 6. überarbeitete Auflage. Seelze: Klett-Kallmeyer.

\section{Dr. Stephanie Moser}

Prof. Dr. Doris Lewalter

Professur für Formelles und Informelles Lernen

Technische Universität München

Arcisstr. 21

80333 München

E-Mail: stephanie.moser@tum.de doris.lewalter@tum.de

\section{Prof. Dr. Henrik Saalbach}

Universität Leipzig

Erziehungswissenschaftliche Fakultät

Marschnerstr. 31

04109 Leipzig

Leipziger Forschungszentrum für

frühkindliche Entwicklung (LFE)

E-Mail: henrik.saalbach@uni-leipzig.de

\section{Prof. Dr. Olaf Köller}

Leibniz-Institut für die Pädagogik der Naturwissenschaften und Mathematik Olshausenstr. 62

$24118 \mathrm{Kiel}$

E-Mail: koeller@leibniz-ipn.de

\section{Prof. Dr. Sabine Walper}

Deutsches Jugendinstitut

Nockherstr. 2

81541 München

E-Mail: walper@dji.de 